\title{
Laser induced surface modification for inkjet printing and coating
}

\author{
Olivera Scheuber, Eleonora Frau, Silvia Schintke*, Member, IEEE \\ Laboratory of Applied NanoSciences, Department of Industrial Technologies, HEIG-VD, HES-SO // University \\ of Applied Sciences Western Switzerland (HES-SO), CH-1401 Yverdon-les-Bains, Switzerland \\ Tel: +41 2455761 67, e-mail: silvia.schintke@heig-vd.ch
}

\begin{abstract}
Lasers are nowadays widely applied in additive manufacturing and several laser based techniques related to inkjet printing are emerging. In this paper, surface treatment of nanoparticle coatings using a commercial laser engraving machine are presented. Experiments were performed on (i) thin thermally cured silver nanoparticle coatings on glass and (ii) iron oxide $\left(\mathrm{Fe}_{2} \mathrm{O}_{3}\right)$ nanoparticle dispersion coatings on glass. The laser treatment on $\mathrm{Ag}$ films illustrates local melting or dewetting behavior dependent on laser power and on the density of engraving patterns. For $\mathrm{Fe}_{2} \mathrm{O}_{3}$ coatings, direct laser writing on dried layers and laser treatment on fluid nanoparticle ink layers are investigated. We demonstrate and discuss in particular the generation of large area laser induced microstructures in vertically confined nanoparticle ink films. Controlled ink accumulation is generated by the laser pulses. Furthermore, 2D porous network structures, as well as laser induced large area filament structures are generated by heat driven capillary flow. Tailored adjustments of nanoparticle inks, film thickness and laser treatment patterns open perspectives for the generation of laser induced self-assemblies, e.g. for novel fabrication processes for 2D metamaterials, for sensor developments or advanced anti-counterfeit applications.
\end{abstract}

Keywords: laser, coating, microstructuring, nanoparticle inks, thermocapillar flow

\section{INTRODUCTION}

Lasers are nowadays widely applied in the fields of additive manufacturing (AM), 2D and 3D printing technologies, e.g. in selective laser melting (SLM), selective laser sintering (SLS), stereolithography, as well as for direct writing or engraving processing [1]. In the field of printed electronics, laser treatment finds furthermore increasing application for the selective curing of nanoparticle (NP) inks [2]. Novel laser assisted inkjet printing and coating techniques for nano- and microstructure generation as well as for advanced inkjet printing using the laser treatment of liquid layers are recently emerging, e.g. laser-induced wet-etching, e.g. [3], or Laser Induced Forward Transfer (LIFT), e.g. [4]. In this paper we study the laser treatment of dried and wet $\mathrm{Fe}_{2} \mathrm{O}_{3}$ NP ink layers, we demonstrate the generation of laser induced large area micro structures by laser treatment applied to vertically confined ink layers.

\section{EXPERIMENTAL}

\subsection{Thin film preparation}

Glass micro slides $(75 \mathrm{~mm}$ x $25 \mathrm{~mm}$, thickness $(1.00 \pm 0.04) \mathrm{mm}$, Sigma Aldrich (Corning)) are used as substrates. Ag thin films on glass were prepared by spincoating of a nanoparticle (NP) suspension and subsequent curing on a hot plate at $160^{\circ} \mathrm{C}$. For $\mathrm{Fe}_{2} \mathrm{O}_{3}$ large area coatings, a NP dispersion (particle size $<100 \mathrm{~nm}$ (DLS), average particle size < $30 \mathrm{~nm}, 20$ wt. \% in $\mathrm{H}_{2} \mathrm{O}(1.17 \mathrm{~g} / \mathrm{mL}$ ) (Sigma Aldrich)) has been used. Coatings have been obtained by blade coating and subsequent drying [5]. The NP suspension has been characterized by dynamic light scattering (DLS, Nanolab 3D system (LS Instruments)), as presented in [5,6]. Wet confined $\mathrm{Fe}_{2} \mathrm{O}_{3}$ films have been obtained by drop casting on a glass substrate, a glass cover slip was then placed on top in order to vertically confine the thin liquid film.

\subsection{Laser treatment and characterization}

For laser treatment, a laser engraving machine (Kkmoon, ed. 2018) was used for all experiments (nominal maximum laser power $3000 \mathrm{~mW}$, nominal laser wavelength $450 \mathrm{~nm}$, pulse duration not specified (reasonably supposed to be in the range of nanoseconds)). A bit map test pattern with horizontal stripes (stripe width 35 pixels $=2.5 \mathrm{~mm}$, each) was generated for the experiments; the stripes of the test pattern exhibit different interline spacings; pattern areas including lines with various horizontal dot densities are used for comparison. Laser treatment is performed line by line (forward / backward scans) on a total surface area of 595x360 pixels resulting in a total treatment area of approx. $28 \mathrm{~mm}$ x $17 \mathrm{~mm}$ (i.e. approx. 47 microns per pixel (approx. $20 \mathrm{dots} / \mathrm{mm}$, which is consistent with the specified engraving precision $(0.05 \mathrm{~mm})$, corresponding to a pixel density of approx. $500 \mathrm{dpi}$ ). All experiments were performed using the discrete engraving mode. Settings of contrast ratio ("253"), power (100\%) engraving depths ("100") were used unless indicated differently. Line patterns are oriented parallel to the x-scan direction. Balsa wood samples were used (at a laser power 20\%) for verification of the focus plane adjustment; samples were placed on a flat glazed ceramic tile as a prevention of fire risks. Operation was performed using additional laser security protection shields. For analysis optical microscopy (LEICA Leica DMILM), as well as with interferometric microscopy and/or 3D profilometry (filmetrics) were used. 


\section{RESULTS AND DISCUSSION}

\subsection{Ag thin films}

Thin Ag films have been treated for parameter and pattern adjustments and for validating the observation of typical phenomena in thin film treatment as a working base. Fig. 1 depicts representative results. Fig. 1a shows line treatments for different line spacings and engraving settings ("contrast ratio" (128 vs 253) and "depths" (80 vs 100); Fig. 1b-1d illustrate the laser induced dewetting phenomenon (compare e.g. [7]) of the thin Ag films upon laser treatment obtained using engraving patterns of various line and spot densities.

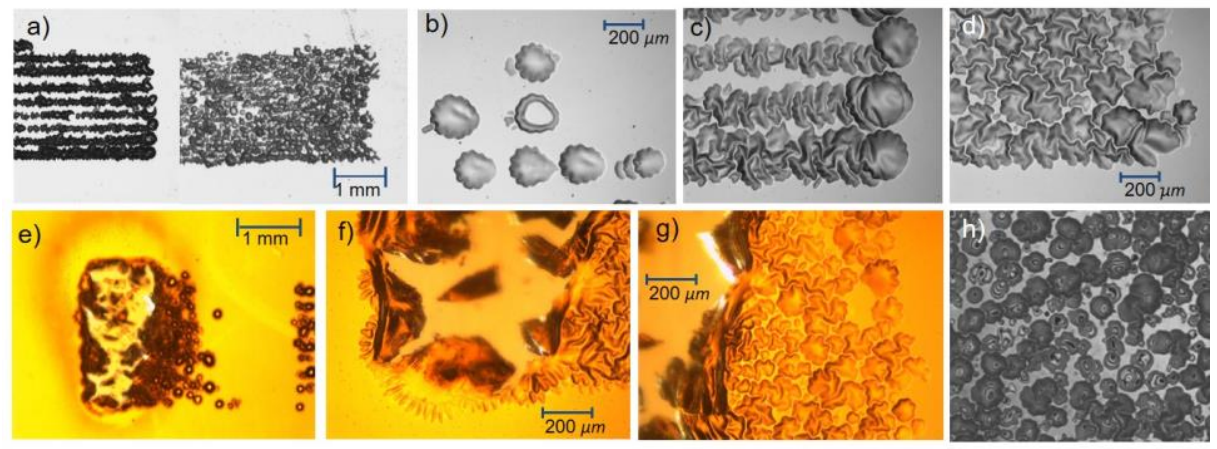

Figure 1. Examples of laser treatment on thin Ag films: a) to g) microscopy images of lines and dots using different line and dot spacings and parameter settings (see text) resulting in typical phenomena on thin metal films, e.g. e) melting, f) thermocapillary effect, g) dewetting. h) interfermometric image of a dewetting zone.

Fig. 1e) (and zooms Fig. 1f and 1g) show local melting of the Ag film, as occurring in a fully treated stripe zone (maximum spot density) near the point of scan direction inversion; thermal impact is thus maximised in this situation. Fig. 1e shows that the surrounding Ag layer is also thermally affected. Small periodic structures are formed at the periphery of the melted zone, spreading away from it (Fig. 1f) and can be associated to thermally driven capillary flow (Marangoni effect). Dewetting is observed in the area of lower spot density, further away from the point of change in scan direction (Fig. 1f, 1g). Figure 1h) shows an interferometric microscope image of a typical dewetting zone. The laser treatment on $\mathrm{Ag}$ thin films thus illustrates successfully typical phenomena expected upon $n s$ pulsed laser treatment on thin metal films. In particular, the controlled dewetting of Ag films can be further used for the generation of plasmonic nano- and microstructures.

\subsection{Dry $\mathrm{Fe}_{2} \mathrm{O}_{3}$ nanoparticle ink films}

Fig 2 shows examples of treated lines and patterns on a dried $\mathrm{Fe}_{2} \mathrm{O}_{3}$ coating, patterns are reliably reproduced. Line spacing and spot density within lines determines as expected the contrast. The surface of dried coatings exhibits domain structures [5], upon laser treatment the optical contrast of these structures is affected (Fig. 2), local surface melting and heat driven flow might be at the origin of the observed surface structures (Fig. 2d-f). Melting of large area was not observed on the dried $\mathrm{Fe}_{2} \mathrm{O}_{3}$ coatings. Further studies e.g. with magnetic force microscopy would allow to investigate whether the laser treatment affects the magnetic properties of the layers.
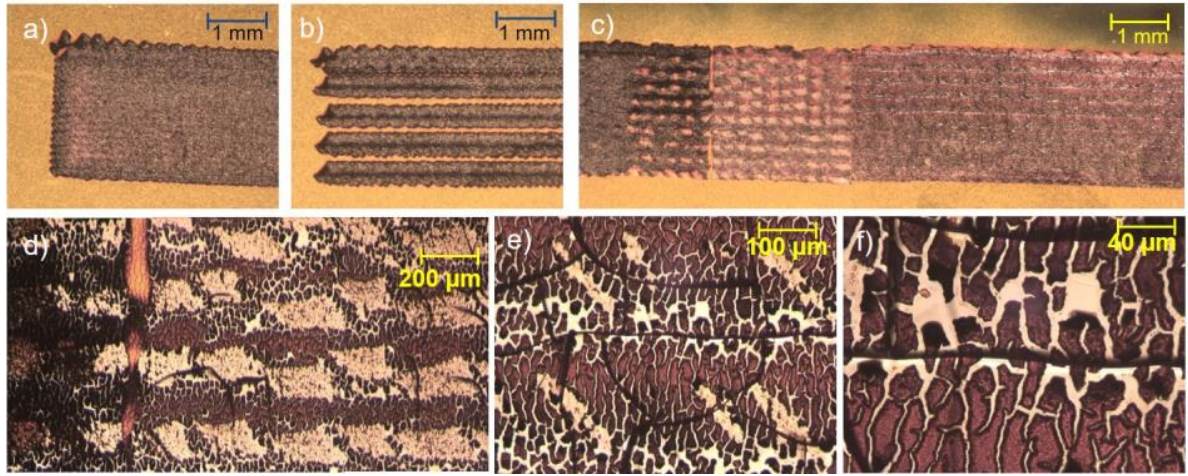

Figure 2. Examples of laser treatment on dried $\mathrm{Fe}_{2} \mathrm{O}_{3} \mathrm{NP}$ ink layers (on glass) by direct laser writing from top.a) and b) structures upon engraving with different line densities; c) stitched image of a laser patterned stripe brighter contrast corresponds to a lower spot density used for patterning; d)-f) zooms of treated area.

\subsection{Wet confined $\mathrm{Fe}_{2} \mathrm{O}_{3}$ nanoparticle ink films}

The laser treatment on wet confined $\mathrm{Fe}_{2} \mathrm{O}_{3}$ NP ink layers (Fig. 3) leads to heat driven flow of the ink. Fig. 3b)-f) shows in optical micrographs upon laser treatment regular patterns of ink accumulation in the shape of wave fronts. The observed two curvatures in different scan lines (e.g. Fig. 3b, c) reflect the two different scan directions. Additional branch formations are furthermore observed (e.g. Fig. 3c,e) within several lines. The 
observed patterns can be explained by heat driven capillary flow, where the ink has dried subsequently. Possibly the laser treatment has in addition led to micro-surface cracks which have preferentially fill with ink. In fact, although the engraving machine is nominally not suited for glass or metal engraving, the presence of the wet ink could possibly lead to laser induced substrate wet-etching [3].

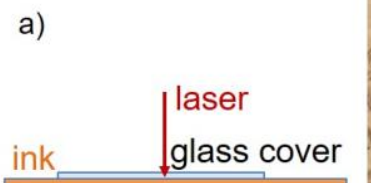

glass substrate

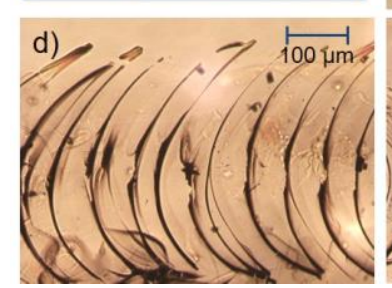

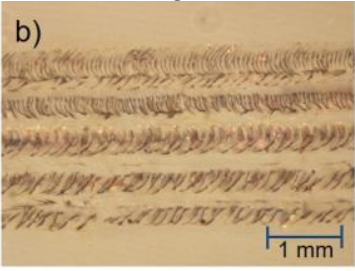

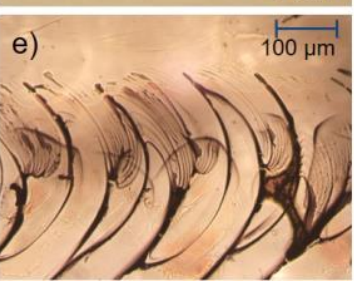

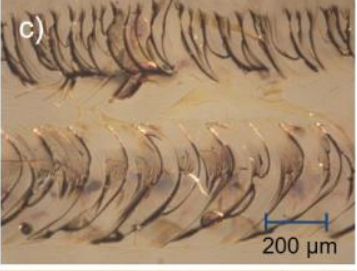

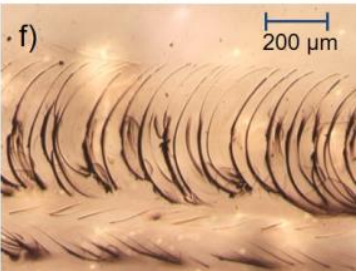

Figure 3. Laser treatment of a confined $\mathrm{Fe}_{2} \mathrm{O}_{3}-\mathrm{NP}$ ink layer: a) set-up for vertical confinement, b) to f) optical micrographs.

In order to further investigate the thermally driven capillary flow and possible wet-etching, layers were treated using stripe patterns with various line and spot densities for comparison. Increasing the line density leads to the observation of highly dense patterns of ink accumulation in wave front patterns in the treated stripe zones (Fig. 4a). The area close to the treated stripes are affected, too (e.g. Fig. 4a in between the treated stripes). Two phenomena are observed in these zones that were not directly exposed to the laser pulses. (i) line filament formation (Fig. 5a) that can be associated to the Marangoni effect and (ii) porous network formation exhibiting residual air bubbles in the film (Fig. 4b-4e). Their formation and shape can be explained by thermally induced perturbation of air bubbles, and subsequent ink drying within the confined space between the substrate and the cover glass. We note, that similar porous structures, but with equilibrium round shapes, can be observed in untreated confined ink layers when tiny air bubbles are incorporated in the ink (not shown here). Porous network ink structures superpose with the wave front structures in case of liquid excess in laser treated area (Fig. 4f, 4g).

Such networks represent conductive percolation structures, thus, mastering their large area fabrication is of interest e.g. as a novel fabrication method for conducting transparent thin films, alternatively to indium based transparent electrodes and as a complementary technique to microstructuring by laser interference [10].
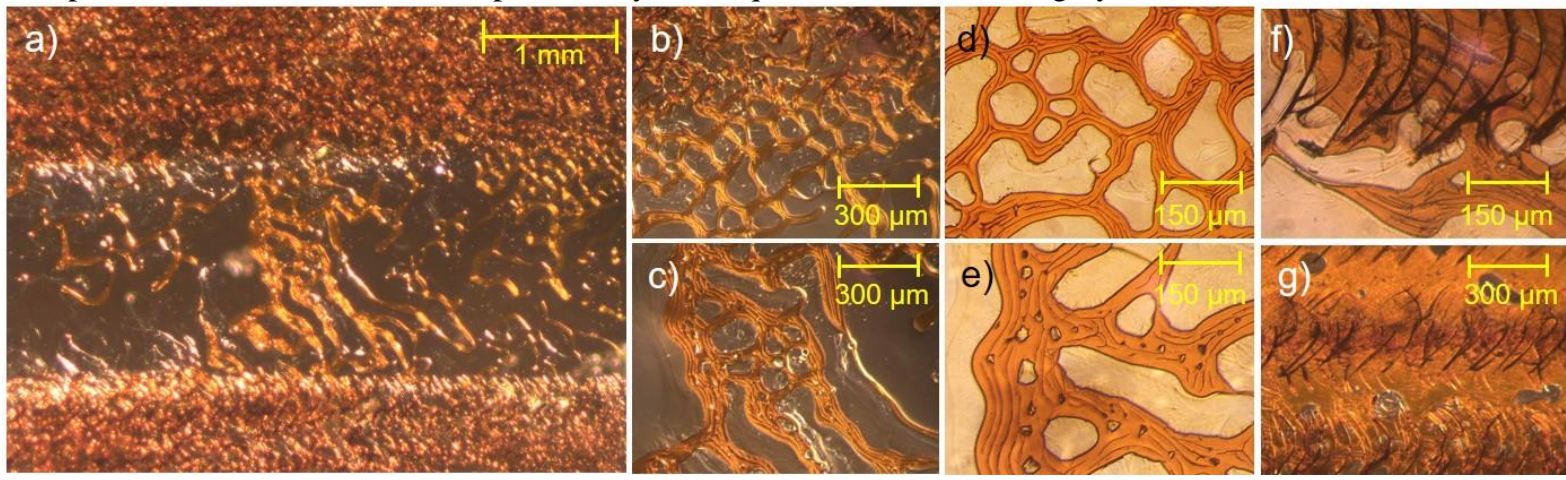

Figure 4. Laser treatment of a confined $\mathrm{Fe}_{2} \mathrm{O}_{3}-\mathrm{NP}$ ink layer, optical micrographs: a) central zone between two treated stripes (top and bottom) with porous network structures of ink formed in the central (non-treated area); b)-e) zooms in central (non treated area) of a); f) g) examples of treated area with excess of inks. Images a)-c) were enlightened from top and bottom during acquisition for better visibility of the structures.

For thin ink films, laser induced formation of large area line filaments, i.e. ink accumulation along long periodic lines are observed in regions close to laser treatments (Fig.5). The laser induced spreading in filament structures can be explained by the Marangoni (thermos-capillary) effect and contact line instabilities with thermally induced surface-tension gradients due to the imposed gradient in temperature [8]. The here observed effect compares to the observation of the spreading of the melted metal film area (Fig 1f), but is leading for the inks to long distance fluid transport and laser induced structuring within the vertically confined film.

The here demonstrated laser induced large area line filament generation is promising for microfluidic applications and cost-efficient microstructure fabrication processes by optical manipulation [8], e.g. for manufacturing processes that aim at metallic, photonic, or magnetic nanoparticle ink structures. 

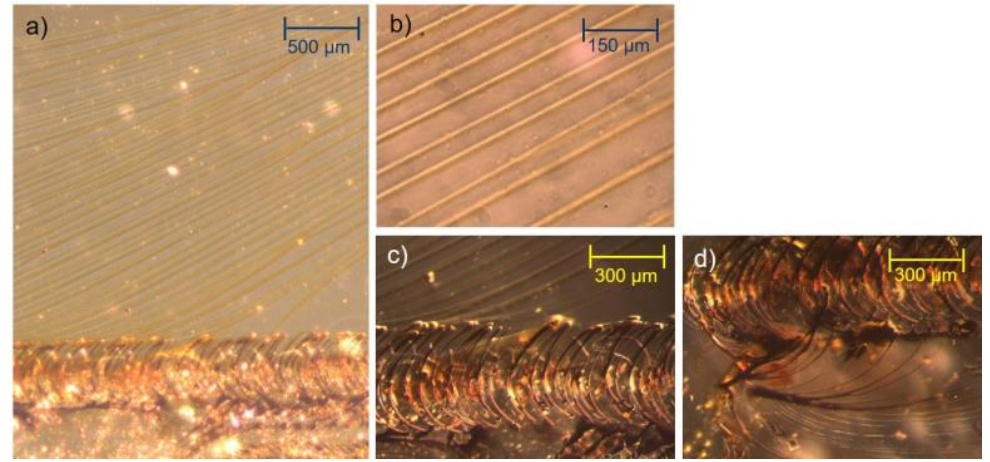

Figure 5. Examples of large area line filament formation in non-treated area by laser induced thermally driven capillary flow.

The application of controlled laser induced periodic structure generation using tailored 2D confined liquid layers is thus of interest for sensor developments, as well as for the fabrication of photonic devices and 2D metamaterials or plasmonic nanostructuring [11].

\section{CONCLUSIONS}

Laser induced microstructuring has successfully been applied using an engraving machine for the laser treatment of thin $\mathrm{Ag}$ films as well as on $\mathrm{Fe}_{2} \mathrm{O}_{3}$ dried and wet NP coatings. In summary we have in particular demonstrated controlled ink accumulation in microstructured wave front patterns and manufacturing of large area laser induced filament line structures by thermocapillary effects in confined fluid layers. The results are promising for microstructuring applications in microfluidic applications as well as for the development of novel cost-efficient manufacturing processes for photonic devices, 2D metamaterials, sensors or anti-counterfeit structures in advanced printing and additive manufacturing for the fields of printed electronics and photonics.

\section{ACKNOWLEDGEMENTS}

This work has been financially supported by the HES-SO project PRONANO (grant number 81265/IA-EXT1757). We kindly acknowledge N. Fosso for the preparation of Ag thin film coatings.

\section{REFERENCES}

[1] Mohammad Vaezi \& Hermann Seitz \& Shoufeng Yang, A review on 3D micro-additive manufacturing technologies, Int. J. Adv. Manuf. Technol. 67 (2013) 1721-1754.

[2] Michael J. Renn, Matthew Schrandt, Jaxon Renn, and James Q. Feng, Localized Laser Sintering of Metal Nanoparticle Inks Printed with Aerosol Jet ${ }^{\circledR}$ Technology for Flexible Electronics, Journal of Microelectronics and Electronic Packaging 14 (2017) 132-139; doi:10.4071/imaps.521797.

[3] M Yu Tsvetkov, V I Yusupov, N V Minaev, P S Timashev, K M Golant, and V N Bagratashvili: Effects of thermo-plasmonics on laser-induced backside wet etching of silicate glass, Laser Phys. Lett. 13 (2016) 106001.

[4] Pere Serra and Alberto Piqué, Laser induced Laser-Induced Forward Transfer: Fundamentals and Applications, Adv.Mater. Technol. 4 (2019) 1800099.

[5] N. Fosso, E. Frau, and S. Schintke: Structuring of magnetic nanoparticle fluids for decorative and functional printing and coating, Transparent Optical Networks (ICTON), 20th International Conference on, pp. 1-4, 2018, IEEE Xplore https://ieeexplore.ieee.org/document/8473656.

[6] E. Frau and S. Schintke: Modulated 3D Cross-Correlation Dynamic Light Scattering of Magnetic Nanoparticle Inks, Transparent Optical Networks (ICTON), 20th International Conference on, pp. 1-4, IEEE, 2018, IEEE Xplore https://ieeexplore.ieee.org/document/8473655.

[7] Harim Oh, Alexander Pyatenko, Myeongkyu Lee: Laser dewetting behaviors of Ag and Au thin films on glass and Si substrates: Experiments and theoretical considerations Appl.Surf.Science 475 (2019) 740-747.

[8] Nicolas Garnier, Roman O. Grigoriev, and Michael F. Schatz, Optical manipulation of Microscale Fluid Flow, Physical Review Letters 9 (2003) 054501-1 and references therein.

[9] Francesc Font, Shahriar Afkhami, Lou Kondic: Substrate melting during laser heating of nanoscale metal films, International Journal of Heat and Mass Transfer 113 (2017) 237-245.

[10] Sebastian Eckhardt, Mathias Siebold, and Andrés Fabian Lasagni, Laser induced microstructures metal thin films as promising alternative for indium based transparent electrodes, Optics Express 24 (2016) A553.

[11] Nikolaos Kalfagiannis, Demosthenes C. Koutsogeorgis, Elefterios Lidorikis and Panos Patsalas, Laser Annealing as a Platform for Plasmonic Nanostructuring, book chapter in Nanoplasmonics -Fundamentals, Ed. Grégory Barbillon, Intechopen 2017, ISBN 978-953-51-3278-3, http://dx.doi.org/ 10.5772/67670. 